\title{
Strategies for Astronomy Development in the Southeast Asia
}

\author{
Boonrucksar Soonthornthum \\ National Astronomical Research Institute of Thailand, \\ Ministry of Science and Technology
}

Astronomy is the science in which we study about everything in the universe, in which we derive the properties of celestial objects, planets, stars, galaxies and so on. These properties deduce the laws by which the universe operates. Astronomy is a remarkable subject which has had a profound effect on the human curiosity and inspiration. So, Astronomy can be used as powerful tool for the development of human resources and human capacity buildings on Science and Technology.

During the past few decades, Astronomy in Southeast Asia has developed significantly. Major astronomical facilities such as optical and radio telescopes have been provided and human resources in Astronomy have been developed and increased. Several countries in Southeast Asia have established their national institutes in Astronomy, Space Science and related disciplines, namely, LAPAN in Indonesia, ANGKASA in Malaysia, PAGASA in Philippines and NARIT in Thailand.

One of the important strategies for developing Astronomy is to use the national institute to drive the national policy in promoting astronomy in the country. The national institute can be a center for the coordination of astronomical activities in schools, universities and research institutes. Fund raising for supporting astronomical activities and the construction of the national astronomical infrastructures can be done through the national institute.

National and international collaborations are also a crucial strategy for the development of Astronomy in Southeast Asia. National institutes can create a national network in Astronomy with universities for the cooperation on research, supporting education and public outreach activities. University staff and researchers can cooperate on some mutual interest research topics with joint publications. University students can access the national astronomical facilities and work under co-supervision of researchers of the national institute. Moreover, the national institute can also get the support from universities and schools in the country for the astronomical outreach activities throughout the country.

International collaboration on Astronomy in Southeast Asia is operated through the Southeast Asia Astronomy Network (SEAAN). SEAAN is a powerful mechanism in nurturing the development of Astronomy in Southeast Asia. Several activities have been done through the SEAAN network especially the promotion of human resource development and human capacity building in Astronomy in Southeast Asia through training. Each year, several schools and workshops in Astronomy on different levels were organized for participants in the Southeast Asian countries. Support and cooperation, including experts and funding, came from many well-established astronomical institutes and observatories outside Southeast Asia.

Recently, the Southeast Asian Regional Office of Astronomy for Development (SEA ROAD) was established in Thailand through the appointment of the Office of Astronomy for Development (OAD) under the International Astronomical Union, (IAU). The operation of SEA ROAD, under the support of the OAD, would be a substantial mechanism 


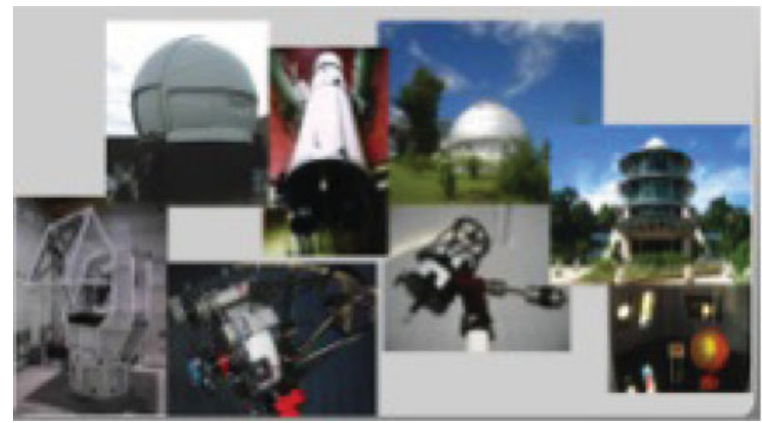

Figure 1. Astronomical infrastructures at the national institutes in Southeast Asia

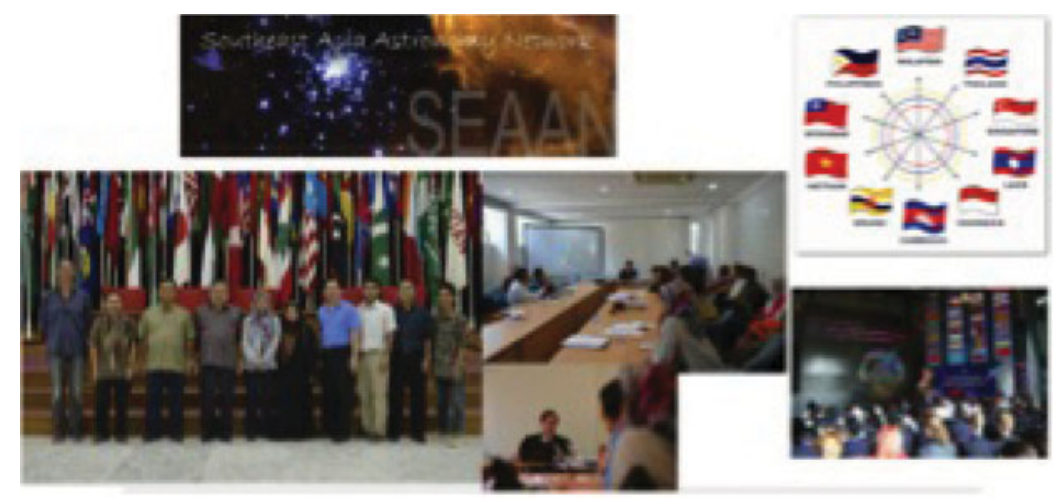

Figure 2. The Southeast Asia Astronomy Network (SEAAN) Meeting

for materializing the cooperation to promote and proliferate Astronomy in Southeast Asia. 